\section{The effect of lesions in caudate nucleus and superior colliculus upon adaptation to prisms in monkey}

\author{
JOSEPH BOSSOM* \\ Massachusetts Institute of Technology, Cambridge, Mass. 02139
}

Monkeys wear prism spectacles for $24 \mathrm{~h}$ in an open-loop situation in which they do not see either their arms or bodies. The main effect of wearing these prisms is a change in the direction of reaching for visually localized objects. However, following surgery, monkeys with bilateral lesions in the head of the caudate nucleus show smaller reaching aftereffects. Monkeys with superior colliculus lesions adapt to prisms as well as they did prior to surgery but show a small amount of past-pointing.

In a previous report, Bossom (1965) described an impairment in visuomotor plasticity in monkeys with bilateral lesions in the head of the caudate nucleus. These monkeys were found to be less able to adjust their reaching to prismatic displacement of the visual image than either normal monkeys or monkeys with other lesions. The purpose of this experiment was to replicate and verify these earlier findings using each animal as its own control.

\section{SUBJECTS}

Eight male Macaca mulatta were used in this experiment. These animals were adolescents, averaging 10-12 lb at the time of testing and surgery.

\section{PROCEDURE}

This method of measuring the accuracy with which monkeys reach for a visual target has been described elsewhere (Bossom \& Ommaya, 1968). The animals were tested in primate restraining chairs. Reaching was measured using a shelf which fit against the monkeys' faces below eye level and which was opaque so that the animal saw neither his arm nor his body during testing. To prevent the animal from viewing his fingertips during reaching, the food appeared only briefly in a small aperture behind an opaque wall; this aperture closed after the reach had been begun but before the hand reached the outer edge of the shelf.

The $S$ obtained food, either a raisin or Spanish peanut, by reaching forward under the shelf and over the far edge where the food had been placed. In so doing, the hand crossed a centimeter ruler affixed to the far surface of the shelf. The distance between the food and the point on the ruler intersected by the radial surface of the hand was the measure of

*This research was supported by NSF Grant GB 7887 and NIH Grant $\mathrm{K}-3 \mathrm{MH}$ 8435 . reaching error. By judicial placement of extra food, the animal was guaranteed a morsel for each reach regardless of its accuracy. He did not, therefore, obtain the same peanut that he had seen and neither were the reaches shaped in any orderly way.

To obtain a stable base, the animals were tested extensively before prism spectacles were placed over their eyes. These prisms (20 diopters) displaced the visual field $11.2 \mathrm{deg}$ and provided a visual field of approximately $60 \mathrm{deg}$. After testing, an opaque collar was added to the chair so that the animals could not see either their arms or bodies during the prism exposure. The testing shelf was then removed, and the animals were exposed in the prisms for $24 \mathrm{~h}$. Under these conditions, the animals were entirely encased except for their heads. This required that the only information that they could obtain be derived from observing visual changes that occurred while they moved their heads. This is a less effective procedure than allowing them to view their bodies, but it eliminates all direct sources of information about the induced disparity between movement and vision (Bossom, 1964). SUMMARY

The surgery was performed under routine aseptic conditions. The monkeys were anesthetized with sodium pentobarbital, shaved, scrubbed, and placed in a head holder. A stereotaxic head holder was used for the dc lesion, and the more flexible NIH monkey head holder was used for superior colliculus ablations. The bone over the area of cortex to be exposed was removed bilaterally with a rongeur in the caudate operations. A replaceable bone flap was removed in the resections of superior colliculus.

The dc lesions in caudate nucleus were made using a 1-mm stainless steel electrode which was fully insulated except for $2 \mathrm{~mm}$ at its tip. A gradually increased current which was brought to $5 \mathrm{~mA}$ was sustained for $2 \mathrm{~min}$ at each coordinate. The coordinates at which burns were made in the head of the caudate nucleus were as follows: 24 anterior (A); 4.5 lateral (L); 11.5 height $(\mathrm{H}) ; 23 \mathrm{~A}, 5 \mathrm{~L}, 10$ and $11.5 \mathrm{H}$; $21 \mathrm{~A}, 4 \mathrm{~L}, 9.5 \mathrm{H}$, and $11.0 \mathrm{H} ; 21 \mathrm{~A}, 6$ $\mathrm{L}, 13 \mathrm{H}$; a total of six burns in the head of each caudate neucleus were made. The aspirated lesions in superior colliculus were placed using a subtentorial approach. The dura was then approximated and sewn closed. HISTOLOGY

The brains were perfused with saline and formalin in the deeply anesthetized animal and removed and kept in formalin solution for 2 weeks. The tissue was then blocked and imbedded in paraffin. Serial sections were obtained at 10 microns, and every 10 th section containing lesioned structures was saved. This tissue was mounted and stained with thionin.

$$
\text { RESULTS }
$$

When the normal animals reached for food placed at the zero position on the shelf, the radial boundary of their hand(s) intersected the scale at a point which averaged $123 \mathrm{~mm}$ for the caudate group and $166 \mathrm{~mm}$ for the superior colliculus group. This difference was not significant. It is consistent with the average hand width of the monkeys we used, which was $3 \mathrm{~cm}$. Following surgery, a small
DECAY OF PRISM-INDUCED MISREACHING AFTER EFFECT

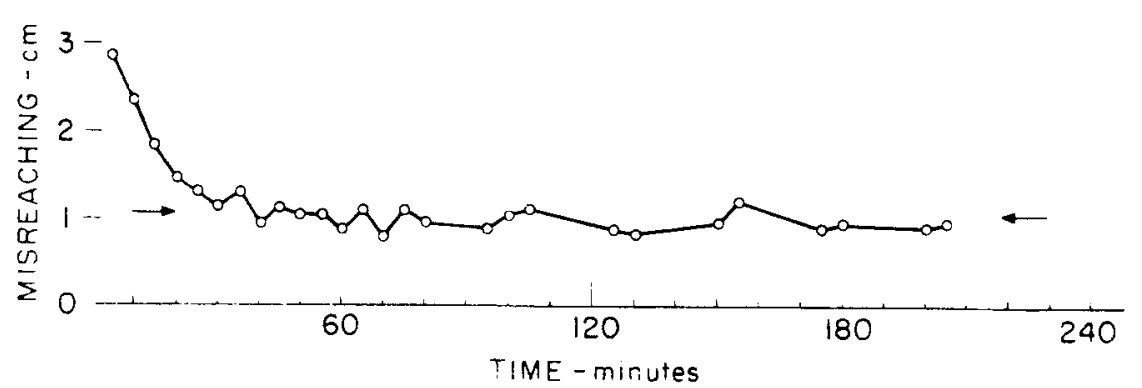

Fig. 1. Decay of prism-induced misreaching aftereffect. 


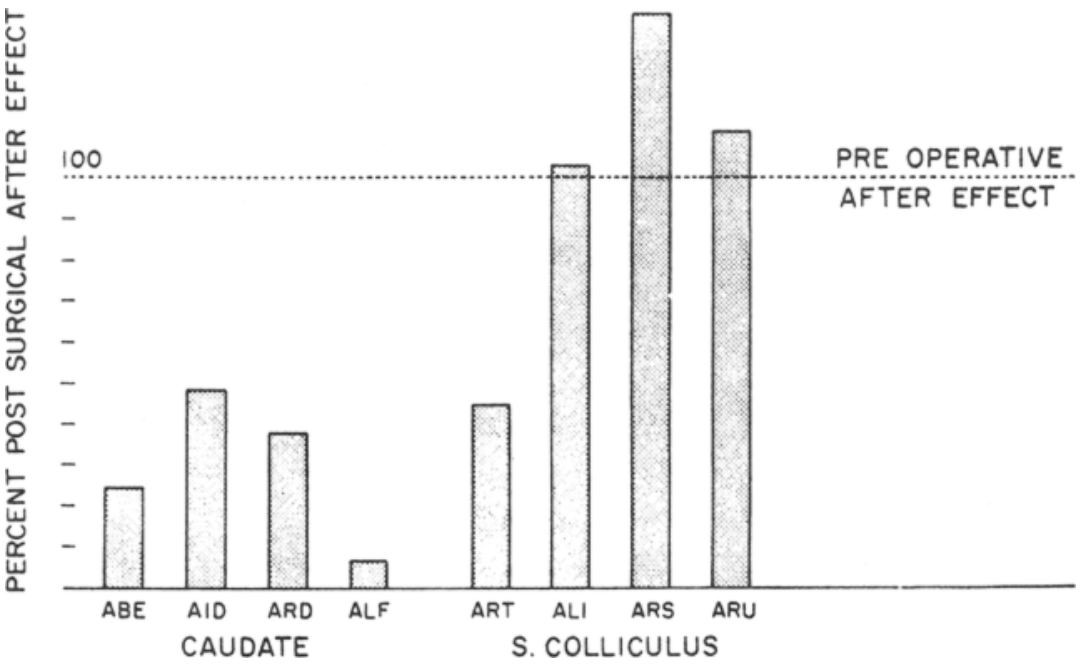

Fig. 2. A comparison of pre- and postsurgical misreaching aftereffects. Animals with lesions in caudate nucleus are significantly impaired.

misreaching error was found in the superior colliculus animals which consisted of reaching wide to the right if the right hand was used or to the left if the left hand was used. The magnitude of this difference was $13 \mathrm{~mm}(\mathrm{p}<.0005)$

In order to determine the course of change in aftereffect over time, two animals which showed larger aftereffects than others were exposed for $24 \mathrm{~h}$ and then, when the prisms were removed, tested for up to $4 \mathrm{~h}$. The obtained curve, shown in Fig. 1, depicts the change in aftereffect during that time. Because maximum reduction occurs during the first 5 min following removal of the prisms, only those measurements obtained during that period were used in the statistical analysis of differences between the caudate and superior colliculus groups.

The mean aftereffect observed in both groups prior to surgery was $245 \mathrm{~mm}$. Following surgery, the animals with bilateral lesions in the head of caudate nucleus were found to have an aftereffect that was diminished by $87 \mathrm{~mm}(\mathrm{p}<.0005)$. By contrast, the superior colliculus group showed an aftereffect that was $.07 \mathrm{~mm}$ smaller than their preexposure aftereffect, a difference that was not significant. The extent of the difference is also shown in Fig. 2. Here the postoperative aftereffect is shown as a percentage of the preoperative aftereffect.

Adapting visually guided arm extensions into space so that errors induced by visual displacement become reduced is a process much studied during the past decade. Depending upon the interests of the investigator, the error correction has been obtained in a variety of ways. The technique used here, that of requiring that the $S$ obtain his information by watching the visual world during his head movements and then measuring the effect of this experience on the direction of his reaching movements, is particularly interesting in that it provides a tool for studying the CNS structures which may be involved in motor-outflow systems.

In order to adapt to prisms under these conditions, the animal must know that he moved and where. He must then correlate that information with visual changes that occurred caudate-nucleus lesions impair this sort of adaptation suggests that this structure and perhaps others in corpus striatum are involved in some aspect of central monitoring of motor outflow. Such a role would be consistent with during the movement. The finding that the observed deficits in movement found in cats with lesions in the head of the caudate nucleus (Liles \& Davis, 1969; Gybels, Meulders, \& Colle, 1967). The exact nature of the role of the caudate in such a system requires further study, but these findings and the report of the dramatic impairment of movement that occurs in previously recovered deafferented monkeys following bilateral lesions of the caudate nucleus suggest that animals so operated are less able to keep track of their own movements by recourse to sources of motor outflow signals (Bossom \& Ommaya, 1968).

The misreaching that was observed in the animals that had lesions in superior colliculus is reminiscent of the past-pointing described by Cowey \& Weiskrantz in monkeys with foveal blindness (1961). Other than a transient nystagmus and apparent blindness in the first few days following surgery, our superior colliculus animals showed no obvious abnormality. It is possible that the cause of the errors that were measured is attributable to a combination of impaired visual acuity and a physiognomic bias. However, further study is required before this phenomenon can be adequately explained.

\section{REFERENCES \\ BOSSOM J. Mechanisms of prism adaptation in monkeys. Psychonomic Science, 1964, 1, 377-378. \\ Bossom, J. The effect of cortical and subcortical lesions on prism-adaptation in monkeys with bilateral dorsal rhiz- otomy Brain, 1968, 91 161-172. \\ B OSSOM Jr., \& OMMAYA, A. K. Visuo-motor adaptation to prismatic transformation of the retinal image in monkeys with bilateral dorsal rhizotomy. Brain, 1968, 91, 161-172. \\ COWEY, A., \& WEISKRANTZ, L. Role of experience in misreaching produced by visual cortex lesions. Nature, 1961, 102, 1319. \\ GYBELS, J., MEULDERS, M., CALLENS, M. \& COLLE, J. Disturbances of visuo-motor in tegration in cats with small lesions of the caudate nucleus. Archives Internationales de Physiologie et de Biochemie, 1967, 74, 2, 283. \\ LILES, S. $\delta_{2}$ DAVIS, G. Permanent athetoid an $\perp$ choreiform movements after small cau'sate lesions in the cat. In G. Crane and $R$. Gardner (Eds.), Psychoimpic drugs and dysfunction of basal ganglia. Washington, D.C: Public Health Service, Publication No. 1938,} 1968. P. 98. 